\title{
Evolution of motor dyscontrol in a case of narcolepsy
}

\author{
Devnani P. ; Ravishankar K. \\ Sleep Disorders Clinic, Department of Neurology and Clinical Neurophysiology, \\ Jaslok Hospital and Research Centre, Mumbai \\ Indian J Sleep Med 2010; 5.3, 95-97
}

\begin{abstract}
We present an interesting case of narcolepsy with parasomnia and RBD in a teenage girl and discuss the development and pathophysiology of motor dyscontrol in narcolepsy.
\end{abstract}

Keywords: MSLT, RBD REM, RWA.

\section{Case report}

A 15 year old female presented with a history of sleep disturbances since the age of six. There was a history of sleep walking in the early half of the night. She developed hypersomnolence since the age of nine. There was difficulty arising from sleep in the mornings and she had to take frequent naps during the day with a consolidated second nap period, late in the afternoon lasting 3-4 hours. She gave a history of vivid dreams imagery and hypnic jerks. She also reported a history of vocalising and violent movements (hittingout episodes) during the night of which she had no recall since the past two years. She denied history of sleep paralysis or cataplexy. She had a history of teeth grinding during the night. She also had a history of migraines, the frequency of which was around two episodes per week and was associated with photo/ phonophobhia. The parents reported that she was angered easily and was oppositional. Her developmental history is normal except for delayed teething.

She denied any symptoms suggestive of restless legs syndrome.

\section{Address for correspondence}

Dr. Devnani P. MD, D, ABSM

Sleep Disorders Clinic, Department of Neurology and Clinical Neurophysiology,

Jaslok Hospital and Research Centre, Mumbai
Clinically no abnormalities were detected. General physical and systemic examination including a detailed neurological examination revealed no abnormalities.

All haematological and biochemical investigations were non-contributory. All radiological investigations including a MRI of the brain were unremarkable. She was subjected to a nocturnal polysomnogram which showed hypersomnia with shortened REM onset latency of 3.5 minutes. There were also episodes of REM sleep without atonia. Bruxism was noted with recurring frequency. There were no findings of sleep disordered breathing. She also underwent a Mean Sleep Latency Test ( MSLT). Mean Sleep Latency was 3.89 minutes. The MSLT was consistent with hypersomnia and evidence of REM intrusion (SOREMs) on two occasions. Though all the features of narcolepsy were not noted in the case, based on the findings noted above, a diagnosis of narcolepsy with parasomia and RBD was made.

\section{Discussion}

The presence of rapid eye movement (REM) sleep without atonia associated with prominent motor behavioral manifestations associated with dreaming during REM sleep is the key feature of a condition called REM sleep behavior disorder (RBD) $)^{1,2,5}$. RBD may occur alone and is then called "idiopathic" RBD. However, it is often associated with other neurological diseases such as lesions of the brainstem, neurodegenerative disease, especially synucleinopathies, and

Indian Journal of Sleep Medicine (IJSM), Vol. 5, No. 3, 2010 


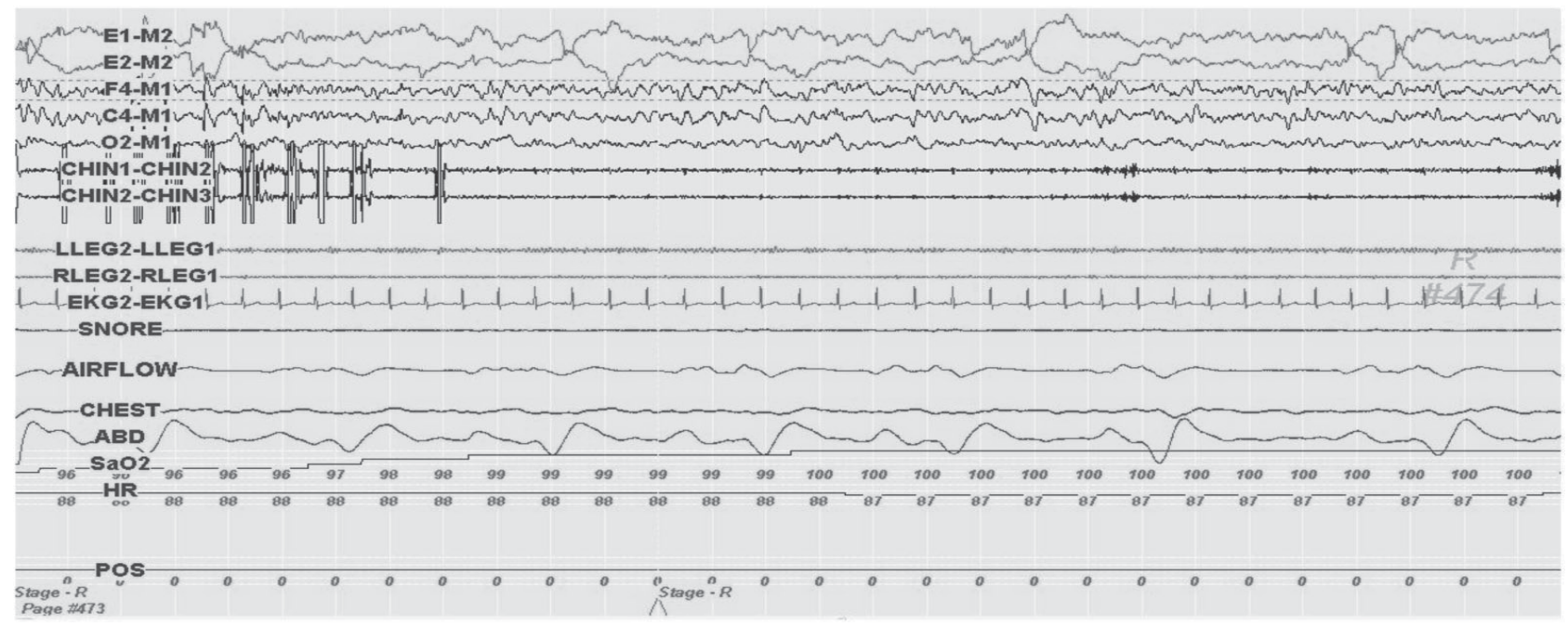

Figure 1: PSG screen showing bruxism with REM atonia

narcolepsy. In these latter cases, it is termed "secondary RBD.”

Polygraphically, tonic and phasic electromyographic activity is evident during REM sleep. When not symptomatic this is called REM sleep without atonia (RWA). The prevalence of RBD in narcolepsy/cataplexy varies from 45\%-61\% with clinical history and PSG detectable in $36 \%-43 \%$ of them. The incidence is higher in patients who have cataplexy as opposed to those without. It is important to note, that RBD can be induced or aggravated by anti-cataplectic treatment (antidepressants). RBD in narcolepsy differs from the idiopathic form because of its much earlier age of onset and different sex ratio ${ }^{4}$.

\section{Understanding REM characteristics of narcolepsy}

Polysomnographic characteristics often differ. Patients with idiopathic RBD tend to have a higher prevalance of RWA and lower REM density than narcoleptic patients.

Quantative analysis of submentalis muscle EMG has demonstrated a difference in the degree of motor dyscontrol between narcolepsy with RBD, idiopathic RBD and RBD with MSA. Another characteristic observed was that in patients of narcolepsy with RBD there was an increase in short-lasting EMG activity from $0-5$ seconds.

Indian Journal of Sleep Medicine (IJSM), Vol. 5, No. 3, 2010

\section{Role of hypocretin}

The loss of hypocretin within the lateral hypothalamus causes narcolepsy in humans.

Recent studies in rats have documented independent pathways in brainstem that mediate the atonia and EEG phenomena of REM sleep. Animal studies suggest that RWA and RBD are two distinct phenomena with different anatomic substrates. Bilateral pontine tegmentum lesions in cats release a state of RWA with minimal motor manifestations. Additional rostro-ventral damage into the midbrain is necessary to release tone completely with elaboration of complex motor behaviours in REM sleep.

Narcoleptics have an increase in REM density contrary to RBD that suggests that different REM components are mediated via independent pathways. Eye movements are generated by the premotor neurons of the reticular formation whereas phasic EMG activity in REM sleep results from phasic depolarisation of alpha motoneurons via both pyramidal and reticular neuron activation ${ }^{3}$.

There is evidence that impaired dopaminergic transmission is an important mechanism in narcolepsy. Different brainstem pathways are associated with different REM phenomena, REM atonia involving the subcoerulus region and EEG phenomena involving preceruleus region.

Patients with narcolepsy tend to have a higher index of PLMS and PLMW as compared to control as seen in patients with RBD. PLMS ratio in REM is noted to be higher than in NREM. However, it is observed that 
motor control in narcolepsy is dysregulated in wakefulness as well with the increase in PLMW index and occurrence of cataplexy, thus, inferring a more global damage of inhibitory systems in narcolepsy.

There are several REM sleep motor dyscontrol mechanisms common to both RBD and narcolepsy. However, behaviour manifestations seem to be less frequent and severe in narcolepsy than in idiopathic RBD. A decrease in hypocretinergic and/or dopaminergic inputs into the brainstem may produce dissociative wake/sleep states.

Retrospective analysis has documented that sleep motor dyscontrol in narcolepsy may begin as a NREM parasomnia and then the onset of narcolepsy may represent the turning point of its intrusion in to REM sleep?

\section{Summary}

Studying the REM characteristics of RBD, narcolepsy and RWA may help in further understanding the pathophysiology and recognising variable clinical manifestations, thus improving patient care. CSF hypocretin levels may play an important role in this regard.

In the above report, the subject presented with a NREM parasomnia and then developed RBD at about the same time as hypersomnia and REM intrusion phenomena set in, she however denied any episode suggestive of cataplexy.

\section{References}

1. Schenck CH, Bundlie SR, Ettinger MG, Mahowald MW. Chronic behavioral disorders of human REM sleep: a new category of parasomnia. Sleep 1986; 9:293-308.

2. Schenck $\mathbf{C H}$, Mahowald MW. Motor dyscontrol in narcolepsy:rapid-eye-movement (REM) sleep without atonia and REM sleep behaviour disorder. Ann Neurol 1992; 32:3-10.

3. Yves Dauvilliers, MD, PhD; Jacques Montplaisir, MD, PhD et al REM Sleep Characteristics in Narcolepsy and REM Sleep Behavior Disorder SLEEP, Vol. 30, No. 7, 2007

4. Carlos H. Schenck MD' and Mark W. Mahowald: REM Sleep Behavior Disorder: Clinical, Developmental, and Neuroscience Perspectives 16 Years After Its Formal Identification in SLEEP. SLEEP, Vol. 25, No. 2, 2002

5. ICSD_International classification of sleep disorders: diagnostic and coding manual. Diagnostic Classification Steering Committee.

6. Schenck CH, Boyd JL, Mahowald MW. A parasomnia overlap disorder involving sleepwalking, sleep terrors, and REM sleep behavior disorder in 33 polysomnographically confirmed cases. Sleep 1997;972-81.

7. Mayer G, Meier-Ewert K. Motor dyscontrol in sleep of narcoleptic patients (a lifelong development?). J Sleep Res $1993 ; 2: 143-8$. 